\title{
DIE BUNDESSTAATLICHKEIT IN NIGERIA
}

\author{
Von Dr. Dieter Schröder
}

Der Ruhm Nigerias als afrikanischer Bundesstaat war bis vor zwei Jahren groß. Nigeria galt als der stabilste und größte Staat Afrikas, der zumindest auf den Westen des unruhigen afrikanischen Kontinents einen beruhigenden Einfluß ausüben könnte. Er wurde als das Gegengewicht gegen den Radikalismus von Staaten wie Ghana und Guinea angesehen, und sein Reichtum und damit die Möglichkeiten $z u$ einer soliden eigenen Entwicklung wurden der Hilfsbedürftigkeit von Staaten wie Dahome oder Niger gegenübergestellt. Solche Hoffnungen, die in der europäischen Literatur weit verbreitet waren, wurden daraus begründet, daß dieser Staat die einzige gesicherte Demokratie in Afrika und der größte und volkreichste Staat des Kontinents wäre. Auch die anderen afrikanischen Staaten haben ihre Unabhängigkeit mit Verfassungen begonnen, die die Demokratie westlichen Verständnisses zum Grundprinzip der staatlichen Ordnung erhoben, aber sie sind heute fast alle Einparteien-Staaten, so daß ihnen eine organisierte politische Opposition, wie sie unserem Verständnis von Demokratie entspricht, abgeht. Von Nigeria wurde angenommen, daß es wider alle Erfahrungen in Afrika seinen Weg als demokratisch geordneter Bundesstaat beharrlich fortsetzen würde und damit zugleich für ganz Afrika Vorbild und Wegweiser für eine bessere Zukunft abgeben könnte. Der letzte Grund für eine solche Annahme war der nigerianische Föderalismus und die Größe des Landes, mit der man die Vorstellung einer soliden Entwicklung verband. Die bundesstaatliche Verfassung wurde als Garantie für die Bewahrung von Größe und Demokratie in unserem Sinne angesehen 1 .

Im Jahre 1966 ist der Bundesstaat Nigeria in eine bisher nicht überwundene Krise geraten. Der „Stabilisator" Westafrikas oder überhaupt Afrikas ist zu einem Faktor der politischen Instabilität geworden. Das Fundament der Stabilität soll die Bundesstaatlichkeit gewesen sein. Wenn man nach den Ursachen der Krise fragt, hat man also das besondere Augenmerk auf die Bundesstaatlichkeit in Nigeria zu richten. Es ist daher geboten, die bundesstaatliche Ordnung Nigerias angesichts ihrer Krise einer kritischen Prüfung zu unterziehen. Zunächst ist dabei die reale Funktion der Verfassung zu untersuchen, insbesondere unter dem Gesichtspunkt, welches Verhältnis die Verfassung zwischen der Gesellschaft und der Staatlichkeit in Nigeria begründen sollte. Dabei ist sowohl die Gesellschaft und ihre innere Ordnung zu untersuchen, da sie den Staat als solchen hervorbringt, aber auch der Staat und seine Verfassung näher zu prüfen, da auch der Staat die Gesellschaft beeinflußt und ihre Anpassung an die staatliche Verfassung betreibt ${ }^{2}$. Wegen dieser Wechselwirkung kann eine Verfassung nicht nach Belieben gesetzt werden, sondern sie hat die Lagen in der Gesellschaft so zu berücksichtigen, daß zwischen Staat und Gesellschaft nicht eine in der Publizistik so beliebte Antithetik entsteht, sondern Staat und Gesellschaft müssen als Aspekte ein und desselben Wesens, nämlich der in einem bestimmten Rahmen zusammenlebenden Menschen und ihrer gegenseitigen Beziehungen, in einem funktionsfähigen Verhältnis zueinander stehen. Für eine bundesstaatliche Verfassung kommt es daher stets darauf an, ob in der Gesellschaft die wesentlichen

\footnotetext{
1 Vgl. z. B. R. L. Watts, New Federation, Experiments in the Commonwealth, Oxford 1966.

2 Zum Verhältnis zwischen Gesellschaft und Staat vgl. insbesondere Herbert Krüger, Allgemeine Staatslehre, Stuttgart 1964, S. 555 ff. und S. 617 ff.; und zur Frage von Gesellschaft und Staat in den Entwicklungsländern vgl. auch Watts, S. 15.
} 
Voraussetzungen einer Bundesstaatlichkeit vorliegen, denn nur dann ist eine solche Verfassung wirksam und notwendig. Und gerade die Notwendigkeit ist es, die die Existenz einer Staatlichkeit bewirkt ${ }^{3}$. Die Notwendigkeit ist geradezu die Logik der Verfassung.

\section{Die vorstaatliche Lage in Nigeria}

Die Ausgangslage des heutigen Staates Nigeria waren die britischen Kolonien um den Niger. In der Mitte des 19. Jahrhunderts hatte Großbritannien auf der Insel Lagos in der Nigermündung die erste Kolonie gegründet ${ }^{4}$. Die Kolonie wurde um das Protektorat Lagos erweitert. Dieses Gebiet wird vorwiegend von dem Stamm der Yoruba bewohnt. Es ist eines der am dichtesten besiedelten Gebiete im ganzen schwarzen Afrika. Seine Besiedlungsdichte entspricht etwa der Besiedlungsdichte Europas im 16. Jahrhundert, während die übrigen Gebiete in der Besiedlung etwa mit den europäischen Verhältnissen um das Jahr $1000 \mathrm{zu}$ vergleichen sind. In dieser Region bestanden daher am ehesten die Lagen, die zur Entwicklung moderner Staatlichkeit in Europa geführt haben ${ }^{5}$. Die Yoruba haben auch schon in vorkolonialer Zeit geordnete Gemeinwesen mit städtischem Charakter ausgebildet, in denen eine hohe afrikanische Kultur entstand ${ }^{6}$. Im Osten an das zunächst von Großbritannien beherrschte Territorium schließt sich das Gebiet von Benin an, dessen Einwohner, teilweise den weiter östlich lebenden Ibos verwandt, eine sehr beachtliche handwerkliche Kunst in Afrika ausgebildet haben?. Diese Zonen bildeten später die Westregion des Bundesstaates Nigeria mit Ausnahme des Bundesterritoriums um die Hauptstadt Lagos. Noch weiter östlich befindet sich das Gebiet der eigentlichen Ibos. Sie wurden von Großbritannien am Ende des 19. Jahrhunderts unter Protektorat genommen, nachdem schon früher einzelne Häuptlinge ihre Stämme unter britischen Schutz gestellt hatten ${ }^{8}$. In diesem sogenannten „OilRiver"-Protektorat war eine politische Organisation der Stämme nicht scharf ausgeprägt, und auf Grund eines verhältnismäßig geringen eigenen kulturellen Standards in dem östlichen Teil des Protektorats in Verbindung mit einer großen Regsamkeit der Einwohner war in dieser Zone die Aufnahme europäischen Gedankenguts sehr groß. Dieser Teil wurde sehr früh und sehr umfassend christlich missioniert; aus seiner Einwohnerschaft rekrutierte die britische Kolonialverwaltung eine Vielzahl von Hilfskräften für die Verwaltung der Besitzung. Die im Norden an die britischen Protektoratsgebiete anschließende Zone umfaßt vor allem das Gebiet der muslimischen Haussa und Fulani, die schon vor der Ankunft der Europäer in Innerafrika zahlreiche große islamische Reiche errichtet hatten, deren bekannteste Sokoto, Kano, Bornu und Kaduna waren ${ }^{9}$. Diese Gebiete waren zunächst von einer britischen Kolonialgesellschaft erschlossen und um 1900 unter britisches Protektorat gestellt worden. Dieses Protektorat wurde im Jahre 1914 mit den Protektoraten an der Küste zum Protektorat Nigeria zusammengefaßt ${ }^{10}$. Die britischen Behörden, vor allem unter der Leitung Lord Lugards, entwickelten gerade in diesen Gebieten

\footnotetext{
3 Vgl. Krüger, a. a. O., S. $25 \mathrm{ff}$.

Vgl. Hellmuth Hecker, Verfassungsregister, Teil IV, Afrika, Asien, Australien, Frankfurt am Main und Berlin, S. $193 \mathrm{ff}$.

s Vgl. Krüger, a. a. O., S. $17 \mathrm{ff}$.

- Vgl. Diedrich Westermann, Geschichte Afrikas, Köln 1952, S. 126 ff. und S. 197.

7 Vgl. Westermann, a. a. O., S. $152 \mathrm{ff}$.

- Vgl. Hecker, S. 196.

- Eine Beschreibung der vorkolonialen polit. Zustände in diesen Reichen findet sich bei Heinrich Barth, Reisen und Entdeckungen in Nord- und Central-Afrika in den Jahren 1849-1855, 2. Bd., Gotha 1857.

10 Vgl. Hecker, S. 195 f.
} 
das Prinzip der indirect rule ${ }^{11}$; sie verzichteten darauf, die in sich stabilen Ordnungen $z u$ verändern und stützten ihren Einfluß vor allem auf eine Zusammenarbeit mit den traditionellen Herrschern. Zwischen den großen Stämmen, manchmal in den Grenzzonen, manchmal in den Siedlungsraum der großen Stämme eingesprengt, lebten mehr als 100 kleine und kleinste Stämme ${ }^{12}$, die sich schon in vorkolonialer Zeit in einem ständigen Abwehrkampf gegen die großen Stämme befanden. Diese kleinen Stämme bilden etwa 40 Prozent der nigerianischen Bevölkerung.

In den Nordgebieten wurde auch in kolonialer Zeit weiterhin Land- und Viehwirtschaft betrieben, während in den Küstenzonen von Lagos her eine Industrialisierung begann, die mit der Entdeckung von Ölvorkommen ein immer größeres Ausmaß annahm. Mit dieser wirtschaftlichen Entwicklung wandelten sich auch die gesellschaftlichen Verhältnisse. Die Stammesordnungen wurden mehr und mehr aufgelöst, modernes Denken trat an die Stelle traditioneller afrikanischer Vorstellungen.

Das Streben nach Unabhängigkeit ging in Nigeria von den Küstengebieten aus, insbesondere von dem östlichen Teil, in dem europäisches Geistesgut den größten Einfluß gewonnen hatte ${ }^{13}$. Der Führer der Unabhängigkeitsbewegung wurde der Ibo Dr. Namili Azikiwe, später der erste afrikanische Gouverneur bzw. Präsident des Bundesstaates Nigeria. Er gründete den National Council of Nigeria and the Camerouns (NCNC) als erste afrikanische Partei in British Nigeria im Jahre 1944 und fand damit bei seinen etwa 8 Millionen Stammesgenossen großen Anhang ${ }^{14}$. Die Partei entwickelte sich sehr schnell $\mathrm{zu}$ einer fast ausschließlichen politischen Institution der Ibos, so daß die Einwohner des westlichen Küstengebietes, die Yoruba, die den Gedanken einer Unabhängigkeit ebenfalls aufgriffen, sich im Jahre 1945 unter dem "Chief" Awolowo enger zusammenschlossen und im Jahre 1950 eine eigene Partei unter dem Namen Action Group (AG) gründeten ${ }^{15}$. Diese Partei stützte sich auf die im westlichen Küstengebiet in einer Zahl von sieben Millionen lebenden Yoruba und anfänglich auch auf die Einwohner des Gebietes um Benin, da sie zunächst dem Tribalismus der Ibos einen von Stammesloyalitäten grundsätzlich freien Regionalismus der Westregion entgegensetzte ${ }^{16}$ und nicht die Entwicklung eines Stammes, sondern einen "afrikanischen Sozialismus“ zum Kern ihres Programms hatte ${ }^{17}$, wodurch sie stets im Gegensatz $\mathrm{zu}$ den im Norden des Landes herrschenden Gruppen stand ${ }^{18}$. Diese Partei forderte auch schon früh die Auflösung der großen Regionen in zahlreiche kleinere Provinzen, um die Bundesgewalt zu stärken ${ }^{19}$. Der Norden blieb von dieser Entwicklung bis in die Mitte der fünfziger Jahre dieses Jahrhunderts unberührt. Es gab dort zwar etwa seit dem Jahre 1944 kleine politische Zirkel und seit dem Jahre 1948 kleine politische Parteien, darunter den im Jahre 1949 als Fortschrittspartei gegründeten Northern Peoples Congress (NPC), aber erst als im Jahre 1951 die traditionellen muslimischen Herrscher erkannten, daß die Entwicklung zur Unabhängigkeit Nigerias nicht mehr aufzuhalten war, wandten sie ihr Interesse den Parteien $\mathrm{zu}^{20}$. Bis dahin sahen sie gerade

11 Vgl. insbesondere Sir F. D. Lugard, The Dual Mandate in British Tropical Africa, Edinburgh 1922.

12 A. H. M Kirk-Greene, The Peoples of Nigeria - The Cultural Background to the Crises, in: African Affairs 1967, S. 3-11, S. 5, nimmt sogar 400 ethnische Gruppen für Nigeria an.

13 Vgl. Walter Schwarz, Tribalsm and Politics in Nigeria, in: The World Today 1966, S. 460-467.

14 Vgl. Ridaard L. Sklar, Nigerian Political Parties, Princeton, N. J., 1963, S. 56.

15 Vgl. Sklar, a. a. O., S. 101.

16 Vgl. Sklar, a. a. O., S. 264.

17 Vgl. Sklar, a. a. O., S. 268.

18 Vgl. John P. Mackintosh, Nigerian Government and Politics - Prelude to the Revolution, Evanstone 1966,

10 Vgl. Sklar, S. 266 ff.

20 Vgl. Sklar, a. a. O., S. $88 \mathrm{ff}$. 
in der britischen Herrschaft eine wertvolle Unterstützung und Ergänzung ihrer eigenen Macht. Sie erhielten zur Sicherung ihrer Herrschaft gegenüber inneren Unruhen die Unterstützung eines großen europäischen Staates, der jedoch im Rahmen der indirect rule auf die innere Entwicklung der Gebiete nur wenig Einfluß nahm ${ }^{21}$, da es ihm an Personal und Mitteln zur vollkommenen Durchdringung des Landes fehlte und er bei der geringen Bedeutung des Gebietes lediglich ein Interesse daran hatte, daß von ihm keine kriegerischen Streifzüge gegen die wichtigen Küstengebiete ausgingen; so daß die kolonialen Handelsbeziehungen ohne Schwierigkeiten abgewickelt werden konnten. Die britische Protektoratsmacht griff in die inneren Verhältnisse selbst in extremen Fällen nur sehr behutsam ein. Sie verbot und bekämpfte zwar energisch den Sklavenhandel und das Fangen von Menschen zum Zwecke der Sklaverei, aber verdeckte Formen der Sklaverei, etwa die Haltung von Haussklaven, die man den Leibeigenen in Europa am ehesten vergleichen kann, konnten auch von energischen britischen Kolonialbeamten bei der Größe und geringen europäischen Durchdringung des Landes nicht beseitigt werden.

Mit Unterstützung des Sardauna von Sokoto, des ranghöchsten unter den muslimischen Herrschern, wurde die NPC nach Revision ihres Programms zur Partei des Nordens, die bei der Einwohnerzahl von 20 Millionen mit der größten Anhängerschaft in Nigeria rechnen konnte. Auch diese Partei - mit dem Motto: "One North: One People Irrespective of Religion, Rank, or Tribe."22 - wollte den Tribalismus zugunsten des Regionalismus verdrängen. Sie stellte dem Drängen der Küstengebiete nach Unabhängigkeit immer wieder entgegen, daß zunächst einmal die Idee „e i n e s Nigeria" festverwurzelt sein müßte, da sonst die Gefahr bestünde, daß der junge Staat im Kampf der Besonderheiten von Regionen und Stämmen zugrunde gehen würde ${ }^{23}$. Trotz dieses verständnisvollen Programms fühlten sich die kleinen Stämme vor allem im Norden durch die großen Parteien bedroht. Sie, mit 40 Prozent der Einwohner Nigerias die größte Gruppe der Bevölkerung des Landes, reagierten auf solche Bedrohungen jedoch nicht mit dem Zusammenschluß zu einer nigerianischen Staatspartei, sondern sie folgten dem tribalistischen bzw. regionalistischen Modell der anderen und gründeten Stammesparteien; sie forderten die Bildung neuer Regionen ${ }^{24}$.

Die Regionen Nigerias unterscheiden sich voneinander in vieler Hinsicht sehr stark. Die Gegensätze in den Religionen, in der inneren Ordnung und in den politischen Auffassungen sind so tiefgreifend, daß nicht nur zur Frage der Unabhängigkeit konträre Standpunkte eingenommen wurden, sondern auch hinsichtlich der Politik eines unabhängigen Staates Nigeria von Anfang an sehr gegensätzliche Ansichten vertreten worden sind. Selbst die außenpolitischen Grundanschauungen sind tiefgespalten. Während der Norden sich sehr stark auch in Zukunft an Großbritannien anlehnen und die Westregion sich mehr der Bündnisfreiheit zuwenden möchte, sind die Ibos vor allem an einer Zusammenarbeit mit den Vereinigten Staaten und an einer eindeutig prowestlich orientierten Politik interessiert. Während der Norden an dem von den traditionalistischen arabischen Staaten propagierten Islam-Pakt interessiert war ${ }^{25}$ und im Bereich des Möglichen den anti-israelischen Kampf der Araber unterstützen will, suchen die Küstenregionen eine Zusammenarbeit mit Israel auf dem Gebiet der Entwicklungshilfe ${ }^{26}$. Bei Erlangung der Unabhängigkeit

21 Vgl. Sklar, a. a. O., S. 323

22 Nach Sklar, a. a. O., S. 326.

23 Vgl. Sklar, a. a. O., S. 98.

25 Vgl. Mackintosh, a. a. O., S. $284 \mathrm{f}$.

26 Vgl. Watts, a. a. O., S. 78. 
bestand in außenpolitischen Fragen lediglich darin Übereinstimmung, daß Nigeria Mitglied der Vereinten Nationen und des Commonwealth, daß es grundsätzlich panafrikanisch orientiert und gegen den Kommunismus sein sollte ${ }^{27}$.

Durch die indirect rule ist ein friedlicher Anpassungsprozeß unter dem Schutz der britischen Kolonialmacht nicht bewirkt worden ${ }^{28}$. In dem Land bestand und besteht daher keine Einigkeit ${ }^{29}$. Das einzige verbindende Element ist eine gegenseitige wirtschaftliche Abhängigkeit ${ }^{30}$, die beim Norden allerdings weitaus stärker ist als beim Süden. Der Norden braucht die Südregion allein schon, um für den Absatz seiner landwirtschaftlichen Produkte den Zugang zum Meer und damit zur Außenwelt zu gewinnen. Aber der Norden benötigt ebenfalls die Finanzkraft des Südens, wenn er sich modernisieren will. Der Süden sieht im Norden einen nahen großen Absatzmarkt für seine industriellen Produkte. Bis zum Beginn des Jahres 1966 befand sich nahezu der gesamte Handel und die technischen Betriebe im Norden Nigerias in der Hand von Einwanderern aus den Küstenregionen, vor allem Ibos ${ }^{31}$. Als um das Jahr 1950 die Unabhängigkeitsbestrebungen so stark geworden waren, daß auch seitens Großbritanniens Überlegungen für eine unabhängige Zukunft der Besitzung angestellt wurden, forderten daher auch vor allem die Ibos, die Einheit Nigerias zu erhalten, wobei sie allerdings einen Einheitsstaat wünschten, in dem die Ibos als Stamm mit der größten Zahl von in Verwaltung und Wirtschaft ausgebildeten Angehörigen die führende Rolle spielen würden. Die andere Gruppe, die besonders energisch für die Erhaltung der Einheit Nigerias eintrat, allerdings im Rahmen eines Bundesstaates, waren die Splitterstämme. Sie fürchteten, bei einer Auflösung Nigerias in drei große Stammesstaaten von den Hauptstämmen jeweils unterdrückt und vernichtet zu werden, und sahen daher in einem Bundesstaat die beste Vorkehrung, die es ihnen ermöglichen würde, durch wechselnde Bündnisse mit den Hauptstämmen ihre Existenz zu erhalten. Im Gegensatz zu diesen Gruppen, die an der Einheit Nigerias stark interessiert waren, war der Norden bereit, eine Auflösung des Landes hinzunehmen, wenn er damit einer Veränderung der inneren Verhältnisse entgehen konnte. Noch im Jahre 1953 ist im Norden die Sezession befürwortet worden ${ }^{32}$.

Im Jahre 1946 wurde von Großbritannien mit der sogenannten Richards-Verfassung $^{33}$ der Versuch auf einen Einheitsstaat gemacht, der jedoch sehr schnell scheiterte und im Jahre 1951 durch eine Verfassung mit bundesstaatlichen Elementen ${ }^{34}$, die MacPherson-Verfassung ${ }^{35}$, ersetzt wurde. Im Jahre 1954 wurde dann eine rein bundesstaatliche Verfassung, die Lyttelton-Verfassung ${ }^{36}$, eingeführt, aus der sich die Verfassung des Bundesstaates Nigeria von 1960 ergab $^{37}$. Den Bundesstaat Nigeria bildeten die Nordregion, die Westregion und Ostregion an der Küste als Gliedstaaten und das Bundesterritorium Lagos. In den Gliedstaaten blieben die alten Strukturen vollständig erhalten. Dem Bund wurde die Zuständigkeit für die Polizei, das Zoll- und Währungswesen, die wichtigsten Teile des Verkehrswesens und der Wirtschaft, insbesondere des Bergwesens, sowie das Hochschulwesen und nach der Unabhängigkeit für die Außenpolitik und die Verteidigung übertragen.

\footnotetext{
27 Vgl. Claude S. Phillips, The Development of Nigerian Foreign Policy, Evanston 1964, S. $20 \mathrm{f}$.

28 Vgl. Watts, a. a. O., S. 82 und S. $86 \mathrm{f}$.

29 Vgl. Watts, a. a. O., S. $28,42,60 \mathrm{f}$.

$30 \mathrm{Vgl}$. Watts, a. a. O., S. $28,49 \mathrm{ff}$.

31 Vgl. Watts, a. a. O., S. 68; Kirk-Greene, a. a. O., S. 7.

32 Vgl. Watts, a. a. O., S. 29.

33 Fundstellen vgl. Hecker, S. 193

${ }^{4} \mathrm{Vgl}$. Watts, a. a. O., S. 14.

*5 Fundstellen vgl. Hecker, S. 194

36 Fundstellen vgl. Hecker, S. 194.

37 Fundstellen vgl. Hecker, S. 194.
} 
Der Bund erhielt als Einnahmen die Zölle sowie die Umsatzsteuer auf den Verkauf von Rohstoffen einschließlich Abgaben auf die Produktion von Rohstoffen ${ }^{38}$. Die Finanzkraft der an sich stark konzipierten Bundesgewalt beruhte vor allem auf den Leistungen der industrialisierten Küstenregionen und nach der planmäßigen Ausbeutung der Erdölvorkommen in erster Linie auf den Leistungen der Ostregion. Der politische Einfluß im Bund lag allerdings bei der Nordregion, die mit der größten Zahl der Einwohner auch die größte Zahl der Wähler hatte, und zwar Wähler, die unter der Kontrolle der traditionellen Herrscher ohne weiteres für die Partei des Nordens, die NPC, stimmten. Gegenüber dieser Region bildeten die beiden Küstenregionen stets eine Minderheit. Als die erste rein aus Afrikanern bestehende nigerianische Regierung eigenverantwortlich gebildet werden sollte, fürchtete daher jede Region und jeder Hauptstamm, daß eine andere Region den entscheidenden Einfluß auf die Zentrale erhalten könnte und damit ihre als unerträglich empfundenen politischen Vorstellungen auf Gesamtnigeria übertragen würde ${ }^{39}$. In dieser Situation schien es die beste Lösung, wenn alle Regionen, d. h. alle drei großen Stammesparteien, sich gleichmäßig an der Regierung des Bundes beteiligten. Der Norden stellte bei seinem bevölkerungsmäßigen Übergewicht den Premierminister und bot den beiden übrigen Parteien eine Koalition an. Aber nur die Ostregion ging auf das Angebot ein und erhielt dafür zunächst die Stelle des Gouverneurs und nach der Ausrufung der Republik im Jahre 1963 die Position des Staatspräsidenten. Die Partei des Westens spaltete sich über die Frage, ob man eine Große Koalition bilden sollte. Die um Benin ansässigen den Ibos verwandten Ibibios waren für eine Koalition, die Yoruba gegen eine Beteiligung an einer solchen. Awolowo, der Gründer der AG und Oppositionsführer in der Bundeslegislative, repräsentierte die koalitionsfeindliche Gruppe der Partei, während der Ministerpräsident der Westregion und stellvertretende Vorsitzende der AG, „Chief“ Akintola, die Koalition befürwortete. Die Bundesregierung unter dem vom Sardauna von Sokoto unterstützten Premier Sir Abubakar Talafa Balewa förderte die Spaltung, indem sie Akintola stützte. Als die Spaltung der Partei vollzogen war, verhängte die Bundesregierung im Jahre 1962 den Ausnahmezustand über die gesamte Westregion und teilte diese in eine Westregion im engeren Sinne und eine Mittelwestregion auf. Akintola gründete jetzt eine neue Partei, die den Namen United Peoples' Party, später Nigerian National Democratic Party (NNDP) erhielt. Er wurde in einer Koalition mit der NCNC Ministerpräsident der neuen Westregion, während in der neuen Mittelwestregion vom Bund ein Adminstrator eingesetzt wurde. Awolowo wurde verhaftet und wegen Hochverrats zu 15 Jahren Gefängnis verurteilt, der Norden schien damit auf dem Wege, seine Herrschaft auf ganz Nigeria auszudehnen, was bei den stark divergierenden politischen Anschauungen zwischen dem Norden und der Küstenzone die Spannungen im Lande verstärken mußte. Als ein Beispiel für die Unterschiede in den politischen Anschauungen sei nur das Verständnis des staatlichen Amtes erwähnt. Die aus dem Norden stammenden Amtsträger sahen gemäß der Tradition ihrer Heimat in ihren Ämtern primär Erwerbsquellen für ihren und ihrer Angehörigen standesgemäßen Unterhalt. Eine so verstandene zielstrebige Nutzung des Amtes erschien den modern denkenden Küstenbewohnern jedoch als „Korruption“ und Ende staatlicher Verwaltung.

s8 Vgl. Oluwole J. Odumosu, The Nigerian Constitution, London und Lagos 1963, S. $393 \mathrm{ff.}$
s9 Vgl. Watts, a. a. O., S. 112 . 
Der Bundesstaat Nigeria wurde also durch die gegenseitige Furcht seiner Teile und das Bestreben, sich gegenseitig nach Möglichkeit zu kontrollieren, zusammengehalten. Es zeigte sich jedoch, daß das Übergewicht des Nordens immer stärker wurde. Bei den Wahlen des Jahres 1964 wurde die Koalition aufgelöst, weil auch die NCNC der Ostregion erkennen mußte, daß sie im Rahmen der Koalition nicht in der Lage war, die Ausdehnung der Herrschaft des Nordens auf ganz Nigeria wirksam zu verhindern. Die Parteien gingen zwei Wahlbündnisse ein ${ }^{40}$, und zwar verband sich die NCNC der Ostregion mit der oppositionellen AG der Westregion und kleineren Oppositionsgruppen, die sich vor allem auf Splitterstämme im Norden und am Rande der Nordregion stützten. Die Verbindung erhielt den progammatischen Namen „Fortschrittliche Allianz“. Die NPC des Nordens ging mit der von ihr geförderten NNDP Akintolas und einigen Oppositionsgruppen, die sich auf Splitterstämme in der Ostregion und am Rande der Ostregion stützten, die „Nationale Allianz" ein. Das Land war damit in zwei Gruppen gespalten, die Haussa- und Fulani-Gruppe im Norden unter der Führung des Sardauna von Sokoto, der den Ministerpräsidenten Balewa nach außen für sich auftreten ließ, und die Ibo-YorubaGruppe im Süden. Die Haussa-Fulani-Gruppe konnte damit rechnen, auf Grund der Macht ihrer Führer etwa zwei Drittel der abgegebenen Stimmen und damit die alleinige Herrschaft in Nigeria zu erhalten. Angesichts dieser für die Fortschrittliche Allianz aussichtslosen Lage verzichteten diese auf eine Beteiligung an den Wahlen, was sie damit begründete, daß die Wahlergebnisse im Norden mit Sicherheit manipuliert werden würden ${ }^{41}$. Die Nordregion, die die Wahlen erwartungsgemäß gewann, begann jetzt ihre Position in Gesamtnigeria auszubauen. Im Jahre 1965 vereinigte die Bundesregierung das zwischenzeitlich verselbständigte Gebiet um Benin wieder mit der Mittelwestregion und setzte Akintola, den Verbündeten der Nordpartei für das gesamte Gebiet dieser Region als Ministerpräsidenten ein. Es kam daraufhin in der Mittelwestregion, die sich als erste einer Unterwerfung durch die Nordregion ausgesetzt wähnte, zu Unruhen, die von der Ostregion nach Kräften unterstützt wurden; am Ende des Jahres 1965 hatte die Bundesgewalt über das Gebiet außerhalb der Hauptstadt Lagos die Kontrolle verloren. Die öffentliche Verwaltung brach zusammen, die Zentralregierung wandte sich jetzt der Unterwerfung der Ostregion zu, deren im Norden lebende Angehörige in großer Zahl die Flucht ergriffen, während sich an der Küste lebende Angehörige der Nordstämme in ihre Heimatgebiete zurückzogen. Schon während dieser Zeit kam es zu Morden und Gewalttaten an Angehörigen anderer Stämme, die Situation ähnelte immer mehr einem Bürgerkrieg ${ }^{42}$. Im Januar 1966 forderte die Bundesregierung den Rücktritt des Befehlshabers der Bundesarmee, des Generals Ironsi, eines Ibo, und entfernte den stellvertretenden Befehlshaber der Bundespolizei und den Polizeikommissar für die Westregion aus ihren Ämtern. Der Eindruck, daß der Norden Gesamtnigeria seiner Herrschaft unterwerfen wollte, verstärkte sich. Junge Offiziere entführten daraufhin den Ministerpräsidenten des Bundes, der dann wenige Tage später tot aufgefunden wurde; der Sardauna von Sokoto und Akintola, sein Gefolgsmann im Süden, wurden erschossen. Die Armee übernahm die Regierungsgewalt ${ }^{43}$, „um Verbrechertum, Unordnung, Korruption und Despotismus zu beenden". Damit war das nigerianische Ziel der ersten Jahre gescheitert, mittels

to Vgl. Mackintosh, a. a. O., S. $576 \mathrm{ff}$.

41 Vgl. dazu Schwarz, a. a. O., S. $464 \mathrm{ff}$; Mackintosh, a. a. O., S. $551 \mathrm{ff}$.

42 Vil. Afrika, Informationen aus Wirtschaft, Politik und Recht, Hamburg 1966, Nr. 9. S. 14, und

Nr. 11, S. 12

4s Vgi. Africa, Research Bulletin, 446, 468. 
einer Verbindung der Gegner in der Regierungsverantwortung eine Unterwerfung ganz Nigerias durch einen der drei Hauptstämme zu verhindern. Es fragt sich jedoch, ob nur diese politische Taktik gescheitert war oder ob sogar die Idee eines nigerianischen Bundesstaates ihr Ende gefunden hatte.

\section{Die Suche nach einer funktionsfähigen Verfassung für Nigeria}

Der Zusammenbruch der Ordnung in Nigeria war eine späte Rechtfertigung für alle diejenigen, die schon um 1955 vor dem Experiment eines nachkolonialen Bundesstaates gewarnt hatten ${ }^{44}$. Aber mit einer solchen Feststellung war es nicht getan. Es kam und kommt darauf an, eine Verfassung $\mathrm{zu}$ finden, die der Lage in Nigeria so angemessen ist, daß sie eine dauerhafte Ordnung und eine gesicherte Entwicklung des Landes ermöglicht. Für diese Aufgabe stellt sich die beschriebene Lage des Landes so dar, daß drei Hauptstämme mit stark divergierenden Interessen ohne eine gemeinsame Idee innerlich unverbunden nebeneinander leben und nur wirtschaftliche Interessen in ganz eng begrenzten Bereichen Beziehungen zwischen ihnen herstellen ${ }^{45}$. Es bietet sich bei dem Streben aller drei Hauptstämme nach Autonomie als Ausweg aus dem nigerianischen Dilemma der Gedanke an, drei oder vier Staaten zu schaffen, die in Bereichen gemeinsamen Interesses eine funktionale Zusammenarbeit pflegen. Der Führer von „Biafra“, der für unabhängig erklärten Ostregion, hatte in diesem Sinne im Sommer 1967 vorgeschlagen, die „souveränen Nachfolgestaaten Nigerias" sollten Eisenbahnen, Fernstraßen, Luftfahrt, See- und Binnenschiffahrt, Seehäfen, Fernmeldewesen, Wetterdienst, eventuell auch eine Zollunion als "Common Services" betreiben, ohne ihre Souveränität einzuschränken. Auch eine gemeinsame Währung sollte bestehen bleiben, jedoch keine gemeinsame Währungspolitik ${ }^{46}$. Mit der Verwirklichung eines solchen Planes würden die Verhältnisse in Nigeria der zwischen den Staaten Ostafrikas seit langem bestehenden Zusammenarbeit entsprechen. Die höchste Stufe wäre dann die Schaffung eines nigerianischen Staatenbundes, der allerdings nur sehr begrenzte Aufgaben haben könnte, die vor allem im Bereich der Wirtschaftsentwicklung liegen würden. Schon für die Außenpolitik ist ein gemeinsamer Nenner. nicht zu finden. Die einzelnen Regionen haben schon lange vor 1965 eine eigene Außenpolitik betrieben und in Großbritannien, dem wichtigsten Partner des außenpolitischen Interesses Nigerias, neben dem Hochkommissar des Bundes in London eigene Generalagenten eingesetzt ${ }^{47}$. Immerhin möchte auch Biafra für die Zukunft grundsätzlich einen gemeinsamen auswärtigen Dienst erhalten ${ }^{48}$. In einem Staatenbund würden die Hauptregionen die wenigen verbleibenden gemeinsamen Angelegenheiten paritätisch regeln können, und damit würde die Furcht gebannt, daß eine Region durch eine andere beherrscht würde. Es würde also einen HaussaFulani-Staat, einen Ibo-Staat und einen Yoruba-Staat, vielleicht auch noch einen kleinen Benin-Staat geben. Es entstünden also vier Stammesstaaten, die immer noch zu den größten Staaten in Afrika zu rechnen wären. Eine solche Lösung läßt jedoch außer Acht, daß in Nigeria die Stämme an den Grenzzonen so ineinandergeschoben sind, daß eine klare Grenzziehung nicht möglich ist. Insbesondere die

\footnotetext{
4 Vgl. Watts, a. a. O., S. 5.

t5 Vgl. Watts, a. a. O., S. 65

46 Vgl. Keesing's Contemporary Archives 22245

17 Vgl. Afrika 1966, Heft 2, S. 14.

4 Vgl. Frankfurter Allgemeine Zeitung vom 30. VIII. 1967.
} 
kleinen Stämme, also etwa 40 Prozent der Bevölkerung, würden bei einer solchen Lösung völlig der Gnade der großen Stämme ausgeliefert sein. Das nigerianische Dilemma würde sich im kleinen innerhalb der Teilstaaten wiederholen, wenn man das Land nicht in 18 kleine Staaten zerschlagen ${ }^{49}$ und nun wirklich "balkanisieren“ wollte.

Die kleinen Stämme sind es daher gerade, die sich einer Aufteilung Nigerias widersetzen. Die Tatsache, daß der heutige Chef der Militärregierung von Nigeria, Oberstleutnant Gowon, Angehöriger des kleinen Stammes der Tiv ist, eines Stammes, der weitgehend christianisiert ist und europäischen Gedanken sehr aufgeschlossen gegenübersteht ${ }^{50}$, ist ein Ausdruck dieser Situation. Die kleinen Stämme unterstützen den harten Kampf gegen die Ostregion, weil sie nur in einem großen Nigeria die Chancen für ihre zukünftige Existenz sehen, und fordern die Wiederherstellung des Bundesstaates, ohne indes eine Garantie für sein Funktionieren anbieten zu können.

Das eigentliche nigerianische Problem ist heute schon mehr der Tribalismus als der Regionalismus, von dem aus bereits die Unabhängigkeit des Landes betrieben worden ist. Dieser Tribalismus stellt die Existenz jedes anderen Stammes in Frage; die Lösung für Nigeria muß demnach in einer Aufhebung des Tribalismus zu suchen sein. Jede Lösung, die an einen Stamm anknüpft, setzt eine Besonderheit absolut und versperrt damit den Weg zur nigerianischen Allgemeinheit als Voraussetzung eines nigerianischen Staates. Die Besonderheiten, die heute in Nigeria vorherrschen, Stämme und Regionen, beruhen auf Bluts- und Ahnenverbindungen, aber auch Religionen, die eine Integration im Zeichen einer Besonderheit ausschließen, $\mathrm{da}$ niemand nachträglich in eine Bluts- oder Ahnengemeinschaft aufgenommen werden kann und die Aufnahme in eine Religionsgemeinschaft erfordert, daß in existenziellen Fragen die eigene Haltung aufgegeben wird. Diese Besonderheiten verstehen sich ihrer Natur nach nicht als territoriale Gegliedertheiten eines Staates, wie es im Bundesstaat sein sollte ${ }^{51}$, sondern in erster Linie als personale Verbundenheit. Sie bilden mithin nicht Glieder eines Staatsgebietes, sondern Gliederungen des Staatsvolkes, im Extrem sogar mehrere miteinander nur lose verbundene Staatsvölker, die mehr zu eigener Staatlichkeit als zu umfassendem allgemeinem Staatsbürgertum drängen. Bei solcher Lage bietet das föderalistische Prinzip keinen Ausweg, sondern verstärkt nur die Tendenz, den Staat aufzulösen. Ein besserer Weg für Nigeria kann nur dort gesucht werden, wo gelöst von allen Besonderheiten etwas Gemeinsames zu finden ist, nämlich im gemeinsamen Tun, heute und für die Zukunft, in dem Bewußtsein des gegenseitigen Aufeinander-angewiesen-Seins und der gemeinsamen Leistung. Genau diese Art der Allgemeinheit ist es, die wir unter Staat verstehen ${ }^{52}$. Nur der Staat, der allein auf das allgemeine Staatsbürgertum und nicht auf Besonderheiten abstellt ${ }^{53}$, ist in der Lage, die sich bekämpfenden Sonderheiten zu überwinden und die Menschen eines Gebietes aus dem gegenseitigen Kampf zu befreien und zu gemeinsamer Entwicklung zu vereinigen. Der Staat existiert nicht aus Bluts- oder Ahnenverwandtschaft oder Religionszugehörigkeit, sondern allein aus der Erkenntnis, daß eine einmal gegebene Lage nur gemeinsam in einem geordneten Verfahren gemeistert werden kann, also nur mit Vernunft; die reine Vernunft ist es, die allein noch aus dem nigerianischen Fiasko

49 Vgl. Kirk-Greene, a. a. O., S. 10.

so Vgl. Afrika 1966, Heft 11, S. 13.

51 Vgl. Krïger, a. a. O., S. 162.

32 Vgl. Krüger, a. a. O., S. $156 \mathrm{ff}$.

$\therefore$ Vgl. Krüger, a. a. O., S. 93. 
eine Lösung aufzeigen kann. Nigeria ist eine Schöpfung europäischer Rationalität gewesen, wie schon der Name - ein englisches Kunstwort - zeigt. Eine solche Schöpfung der Ratio bedarf für ihre dauerhafte Existenz einer rationalen Ordnung, die die Stämme ihrer Natur nach nicht hervorbringen können.

Die Idee eines nigerianischen Bundesstaates zielte darauf $a b$, die Besonderheiten in den Regionen, in denen sie vorherrschten, zu erhalten und dem Wohl des Ganzen dienstbar zu machen. Aber dieser Weg konnte nicht gelingen, da die Besonderheiten sich in Nigeria so absolut verstehen, daß zwischen ihnen - abgesehen von geringen wirtschaftlichen Interessen - keine Gemeinsamkeit, auf der ein Gemeinwohl aufbauen könnte, besteht. Nigeria war ein Bundesstaat ohne eine positive Bundesidee als Staatsidee. Seine Staatsidee war lediglich die gegenseitige Furcht. Der Weg Nigerias kann also nur die Schaffung eines Staates sein, der ganz bewußt alle regionalen Besonderheiten ignoriert und ihnen ein nigerianisches Gemeinwohl entgegenstellt. Damit allerdings steht Nigeria vor dem Problem nahezu aller jungen Staaten, daß es mangels eines nigerianischen Volkes heute noch nicht möglich ist, ein nigerianisches Gemeinwohl als Richtschnur nigerianischen staatlichen Handelns ${ }^{54}$ festzustellen. Nur einzelne Gruppen der Bevölkerung sind heute auf eine nigerianische Staatlichkeit orientiert, vor allem die Beamtenschaft und die Soldaten, die Gruppen also, die für die Wahrnehmung ihrer Funktion besonders auf moderne, rational geordnete Verhältnisse angewiesen sind ${ }^{55}$. Gerade von diesen Gruppen ist auch der erste Staatsstreich in Nigeria getragen worden.

Es ist anzunehmen, daß General Ironsi, der im Sommer 1966 ermordete Chef der nigerianischen Militärregierung, mit Unterstützung der Mehrheit der nigerianischen Beamtenschaft beabsichtigt hatte, die Besonderheiten der Stämme rücksichtslos an die Seite zu schieben ${ }^{56}$. Er proklamierte am 24. Mai 1966, daß die Bundesverfassung aufgehoben und Nigeria ein Einheitsstaat geworden sei ${ }^{57}$. Um Nigeria zu einem Einheitsstaat machen zu können, bedurfte es insbesondere einer Vereinheitlichung der Verhältnisse im ganzen Lande. Während an der Küste das Leben so verdichtet ist, daß schon in vorkolonialer Zeit in dieser Zone die Verhältnisse nach Verstaatung drängten, sind die vorstaatlichen Verfassungen der Gesellschaft im Norden noch durchaus lebensfähig. Ein Einheitsstaat kann sich mit dieser Feststellung jedoch nicht begnügen, er muß die Verhältnisse mit Macht modernisieren, wenn alle Gebiete in gleicher Weise Staatsgebiet sein sollen. Einer solchen notwendigen Unterwerfung im Einheitsstaat mußte sich der Norden nach Kräften widersetzen. Ironsi setzte die Pläne zur Aufhebung der Regionen und zur Aufteilung des Landes in 35 kleine Provinzen auch nur sehr langsam durch ${ }^{58}$. Er nutzte insbesondere nicht die anfängliche Lähmung des Nordens, dessen Führung ausgeschaltet war, für eine rasche Umwandlung des Landes. Für ihn war allerdings auch ein Hindernis, daß er ein Ibo war und die anderen Regionen befürchten mußten, in einem nigerianischen Einheitsstaat würde der alte Wunsch der Ibos nach Herrschaft über ganz Nigeria verwirklicht werden. Ironsi wurde von Soldaten aus dem Norden ermordet ${ }^{59}$. Sein Plan war vielleicht zu radikal gewesen, aber die Aufgabe, die er für Nigeria erkannt hatte, besteht nach wie vor.

\footnotetext{
5s Vgl. Krüger, a. a. O., S. 208.

55 Vgl. Pye, Lucian W., Armies in the Process of Political Modernization, in: Johnson, Join J., The Role of the Military in Underdeveloped Countries, Princeton, N. J., 1962, S. 69-89, S. 73 if.

si Vgl. Kirk-Greene, a. a. O., S. 4.

57 Vgl. Africa Research Bulletin 534; O. G. v. 24. 5. 1966.

58 Vgl. Schwarz, a. a. O., S. 466; Atrika 1966, Heft 6, S. 9, und Heft 9, S. 13.

50 Vgl. Schwarz, a. a. O., S. 466; Africa Research Bulletin 550.
} 
Ironsis Nachfolger, Gowon, hat den Bundesstaat sofort wiederhergestellt ${ }^{60}$. Die Ostregion hat sich aber bis heute seiner Herrschaft widersetzt und sich im Jahre 1967 als Republik Biafra abgespalten ${ }^{61}$, und auch das Gebiet um Benin hat sich zeitweilig als Republik Benin verselbständigt ${ }^{62}$. Nigeria steht nach wie vor vor der Aufgabe, eine Lösung zu finden, die die Besonderheiten integriert, sie aber so bändigt, daß sich über ihnen eine positive nigerianische Gemeinsamkeit, ein nigerianisches Gemeinwohl entwickeln kann. Die erste Voraussetzung dafür ist allerdings, daß in Nigeria eine führende Schicht von $\mathrm{Nige}$ rian e r $\mathrm{n}$ entsteht, die sich in erster Linie als Bürger dieses Staates und nicht mehr als Ibos, Haussas usw. verstehen. Der Kern für eine solche führende Schicht dürfte in den kleinen Splitterstämmen zu suchen sein, die als einzige Gruppe in der Bevölkerung Nigerias in dem großen Nigeria ein Positivum sehen, und zwar den einzigen und daher notwendigen Schutz vor einer Überfremdung durch andere Stämme, und denen der Stamm angesichts seiner Schwäche, sich unter den anderen Stämmen zu behaupten, belanglos werden müßte. Bei den Tiv, einem dieser kleinen Stämme, läßt sich jedenfalls heute am ehesten Verständnis für die Idee eines nigerianischen Gemeinwohls feststellen $^{63}$, wenn auch hier die Bundesregierung neuerdings auf Widerstand treffen soll64. Die Gruppe der kleinen Stämme würde mit ihren 40 Prozent der nigerianischen Bevölkerung die stärkste Gruppe der Bevölkerung bilden. Gegenwärtig kommt die Mehrheit der Beamtenschaft des Bundes aus solchen kleinen Stämmen, sie haben heute die Macht im Bund ${ }^{65}$. Allerdings bilden sie noch keine Gruppe in der Bevölkerung, die das Schicksal Nigerias allein in die Hand nehmen kann. Sie sind stets auf die Unterstützung mindestens eines der großen Stämme angewiesen. Soweit es heute zu erkennen ist, stützen sie sich gegenwärtig auf den Norden und seine militärischen Einheiten. Damit bringen sie allerdings wieder den Faktor in die nigerianische Politik, an dessen Machtanspruch der nigerianische Bundesstaat gescheitert ist. Mit diesem Verbündeten führen sie Elemente in die Auseinandersetzung hinein, die nicht nur in Nigeria, sondern in der ganzen Welt wegen ihrer archaischen Grausamkeiten Abscheu erregen müssen und deren Auftreten gerade den Widerstand der Ibos gegen ein großes Nigeria verstärken muß. Weitaus erfolgversprechender scheint die Wiederbelebung einer alten Verbindung der kleinen Stämme mit den Yoruba ${ }^{66}$, dem Teil der nigerianischen Bevölkerung, der am weitesten auf dem Wege zur Verstaatung vorangeschritten ist und dem die Idee eines Gemeinwesens und eines nigerianischen Gemeinwohles am ehesten einsichtig sein dürfte. „Chief“ Awolowo, der Repräsentant der Yoruba, war einer der ersten, die die Problematik des nigerianischen Bundesstaates erkannt hatten. Er hat sich konsequent für seine Auffassung eingesetzt. Er hat heute in seiner Programmschrift für eine neue nigerianische Verfassung den Weg Nigerias zur Staatlichkeit am deutlichsten herausgearbeitet. Gegen eine starke Beteiligung der Ibos an einem neuen nigerianischen Staat spricht, daß eine Begeisterung dieses Stammes für eine neue Verfassung und für einen neuen nigerianischen Staat den Argwohn des Nordens wecken wird. Nord- und Ostregion als solche haben in Nigeria also die Chance vertan, bei der Gestaltung der Zukunft des Landes mitzuwirken. Sie müssen als bestimmende Faktoren aus dem politischen Spiel im Lande ausgeschlossen werden.

\footnotetext{
to Dekret Nr. 59, in O. G. v. 1. 9. 1966; Africa Research Bulletin 590.

61 Text der Proklamation, in: International Legal Materials 1967, S. 665 ff

62 Vgl. Africa Research Bulletin $865 \mathrm{~A}$.

63 Vgl. Mackintosh, a. a. O., S. 467.

os Vgl. Frankfurter Allgemeine Zeitung vom 17. 11. 1967

o5 Vgl. Kirk-Greene, a. a. O., S. $8 \mathrm{f}$.

of Vgl. Sklar, a. a. O., S. 348; Mackintosh, a. a. O., S. 463.
} 
Eine Lösung für Nigeria wird nicht um den von Ironsi angedeuteten Schritt herumkommen, die großen Stämme und Regionen stärker aufzulösen, um sie besser gegeneinander stabilisieren zu können. Den Anfang hat die erste Militärregierung mit dem Verbot aller Parteien gemacht, wodurch das institutionalisierte Stammes- bzw. Regionalinteresse vorläufig zerstört werden sollte. Jetzt kommt es jedoch darauf an, zwischen dem Extrem des Einheitsstaates, der eine rasche Vereinheitlichung der Verhältnisse in allen Regionen erzwingt, und dem Extrem der alten nigerianischen Bundesverfassung, die nicht verhindern konnte, daß sich die Besonderheiten der Regionen absolut verstanden, einen Ausweg zu suchen, und zwar in einer Verfassung, die es zuläßt, daß sehr verschiedene Lagen in einem Staat bestehen und schrittweise aneinander angepaßt werden. Das Modell einer solchen Verfassung könnte vielleicht diejenige des Zweiten Deutschen Reiches vom Jahre 1871 sein, unter der verstaatete Demokratie in den Hansestädten und ständische Feudalordnung in Mecklenburg vereint waren und in der keine extreme Einheit so groß war, daß sie allein den Gesamtstaat beherrschen konnte. Es wäre auch an die Schweizer Bundesverfassung zu denken, die sehr unterschiedliche Gliedstaaten wie Genf und den Tessin in einem Staat verbindet. Eine solche Verfassung würde allerdings den Gesamtstaat auf wenige, vor allem nach außen gerichtete Aktivitäten beschränken müssen, und damit einem Staatenbund vielleicht näherkommen als einem Bundesstaat. Die heutige Militärregierung ist in dieser Richtung weitergegangen und hat im Mai 1967 das Land in zwölf Provinzen aufgeliedert, wie schon von ausländischen Beobachtern früher vorgeschlagen worden war ${ }^{67}$, auch nigerianische Politiker hatten schon lange vor Erlangung der Unabhängigkeit Nigerias eine Neugliederung als Voraussetzung innerer Stabilität gefordert. Großbritannien hatte jedoch erklärt, daß die Verwirklichung solcher Pläne eine Verschiebung des für die Unabhängigkeit festgesetzten Termins um zwei Jahre erfordern würde. Das hatten die nigerianischen Politiker wieder nicht gewollt ${ }^{68}$. Dem Antikolonialismus zuliebe hatte man also den jungen Staat bewußt mit einer sehr problematischen Verfassung belastet. Die von der jetzigen Militärregierung eingeteilten Provinzen sollen wieder zu einem Bundesstaat vereinigt werden, der allerdings die Besonderheiten weiter zurückdrängen muß, wenn er das Fiasko des ersten nigerianischen Bundesstaates vermeiden will. Er muß das Allgemeine klar über das Besondere setzen und das Besondere der Kontrolle durch die Allgemeinheit, den nigerianischen Staat, unterwerfen. Eine solche Verfassung dürfte jedoch kaum noch europäischen Vorstellungen von Bundesstaatlichkeit entsprechen. General Gowon hat auch bereits angekündigt, daß es sich um ,an entirely new arrangement, peculiar to Nigeria, which has not yet found its way into any political dictionary" handeln würde ${ }^{69}$. Es kommt in Nigeria in erster Linie auf die Staatlichkeit und erst danach auf den Föderalismus an. Das Modell einer solchen "nigerianischen Bundesverfassung" kann vielleicht Kamerun abgeben, wobei allerdings zu bedenken ist, daß in Kamerun die Stämme schon naturgemäß sehr viel kleiner sind und damit die Gefahr der Vorherrschaft eines Stammes ausgeschlossen ist.

Das Modell eines „afrikanischen Bundesstaates“, wie es in Kamerun entwickelt worden ist ${ }^{70}$, basiert darauf, daß alle Besonderheiten funktional ganz eng mit der Hervorbringung des Allgemeinen verbunden werden, daß sie sich in erster Linie als

\footnotetext{
$67 \mathrm{Vgl}$. Gwendolen M. Carter, National Unity and Regionalism in Eight African States, Ithaca, N. Y., 1966, S. 542; Kirk-Greene, a. a. O., S. 10.

68 The Struggle for One Nigeria, herausgegeben von der Bundesrepublik Nigeria, Lagos o. J., S. 25.

69 Nach Kirk-Greene, S. 10.

70 Vgl. dazu ausführlich Dieter Schröder, Kamerun - Modell eines afrikanischen Bundesstaates?, in: Afrika Spectrum 1/67, S. 5-14, S. 9 ff.'
} 
Variationen des Allgemeinen verstehen und nicht mehr als Besonderheiten. In Kamerun hat der Staatspräsident zugleich die Funktion eines Präsidenten der Regionen und er verkündet als solcher alle gesetzgebenden Akte der Regionen. Die Volksvertreter treten stets als gemeinsames Parlament zusammen, und nur wenn der Staatspräsident es für geboten hält, können sie in partes abstimmen und damit als Vertreter der Besonderheiten auftreten. Die Verwaltung aller Regionen ist dem Bund unterstellt, der sie durch Bundes-Kommissare leitet. Den Regionen sind nur die Kompetenzen überlassen, die aus ihrer Natur heraus zur sachgerechten Lösung genauer Kenntnis der Lage in den berührten Gebieten bedürfen. Damit ist für unsere Vorstellungen ein dezentralisierter Einheitsstaat im Gewande eines Bundesstaates entstanden. Aber allein dieses Modell kann aus dem Kampf der großen Stämme gegeneinander herausführen, indem es den Staat, der sich gegenüber den Besonderheiten neutral stellt, so stärkt, daß das Gemeinwohl sich gegenüber den Sonderinteressen durchsetzen kann. Ob auch Nigeria den Weg in eine "nigerianische Verfassung" findet, wird die Zukunft zeigen. Die Verwirklichung der neuen Pläne kommt jedenfalls nur sehr langsam voran. Beispielhaft dürfte das Verhalten der Nordregion sein. Der regionale Befehlshaber hat im August 1967 verkündet, daß sie im März 1968 in die vorgesehenen sechs Provinzen aufgelöst werden wird ${ }^{71}$, worin nicht gerade eine Begeisterung für die Erneuerung Nigerias zum Ausdruck gelangt.

In Nigeria ist nicht der Föderalismus in eine Krise geraten, sondern man hat hier gemeint, die vielleicht komplizierteste staatliche Verfassung ${ }^{72}$ sei gerade gut genug, um eine Gesellschaft aus vorstaatlicher personaler Ordnung zu moderner Staatlichkeit, d. h. territorialer Allgemeinheit, zu führen. Nigeria hat bewiesen, daß ein solcher Weg jedenfalls nicht beschritten werden kann.

“ Vgl. Keesing's Contemporary Archives 22245.

72 Vgl. Krüger, a. a. O., S. $161 \mathrm{f}$. 